\title{
Numerical Investigation to Fuzzy Volterra Integro-Differential Equations via Residual Power Series Method
}

\author{
Mohammad Alshammari ${ }^{{ }^{*}}$, Mohammed Al-Smadi ${ }^{2}$, Ishak Hashim ${ }^{1}$ and Mohd Almie Alias ${ }^{1}$ \\ ${ }^{1}$ Department of Mathematical Sciences, Faculty of Science and Technology, Universiti \\ Kebangsaan Malaysia, 43600 UKM Bangi, Selangor, Malaysia \\ ${ }^{2}$ Department of Applied Science, Ajloun College, Al-Balqa Applied University, Ajloun 26816, Jordan
}

\begin{abstract}
In this paper, a study of a numerical approximate solution to fuzzy Volterra integro-differential equations is presented under strongly generalised differentiability by applying an influent effective technique, called the Residual Power Series (RPS) method. The solution approach can be expressed on Taylor's series formula in terms of elementary $\sigma$-level representation, whereas the coefficients can be computed by utilising its residual functions. Furthermore, a numerical computational example is given to test and validate the proposed method. The results reached show several features concerning the RPS method such as potentiality, generality and superiority to handle many problems arising in physics and engineering.
\end{abstract}

Keywords: fuzzy IVPs; integro-differential equation; residual power series method; Hukuhara differentiability

\section{INTRODUCTION}

In recent years, the topic of fuzzy integro-differential equations (IDEs) has gained more attention by many scholars for its essential role in modelling many phenomena that arise in various applications in physics and engineering (Gumah et al., 2016; Abu Arqub et al., 2016; Arqub et al., 2017). Several scholars in scientific areas widely use the fuzzy IDEs to understand the structure and predict the behaviour of solutions to the issues under study. Typically, during the formulation of many problems, the resulting information will be subject to uncertainty for various reasons such as errors in the measurement process, inaccurate prediction, collection of erroneous data, as well as bad estimation when determining the initial guesses. Therefore, effective mathematical tools are needed to understand this uncertainty. Consequently, the theory of fuzzy set is a powerful tool for handling and modelling such issues under uncertainty. For other numerical techniques about integro-differential equations, we refer to (Abu Arqub \& Al-Smadi, 2018; Al-Smadi \& Arqub, 2019; Saadeh et al., 2016; Abu Arqub \& Al-Smadi, 2018).

On another aspect, investigations of numerical solutions for fuzzy IDEs are missing and rare. In (Abbasbandy \& Hashemi, 2012; Abbasbandy \& Hashemi, 2010), the authors relied on the method of homotopy analysis and the variational iteration method to find a numerical solution of such equations. The reproducing kernel algorithm was applied for solving a class of fuzzy Fredholm-Volterra integro-differential equations (Abu Arqub, 2017). Haar wavelet method was used to solve a class of fuzzy Volterra integro-differential equations (Altawallbeh et al., 2018). In the current paper, we extend the application of the residual power series (RPS) method to investigate the approximate analytic solutions of fuzzy Volterra IDEs under generalised H-differentiability (AbuGdairi et al., 2015; Al-Smadi et al., 2017; Al-smadi, 2019; Abu Arqub et al., 2018; Al-Smadi, 2018; Freihet et al., 2019).

The skeleton of this article is structured as follows: In section 2, some basic definitions and preliminary facts related to fuzzy calculus are given. The fuzzy Volterra integro-differential equations are discussed in section 3 . In section 4, a description of the RPS method is presented. One numerical example is given to illustrate the RPS method. Finally, a summary of this paper has been provided. 


\section{PRELIMINARIES}

The material in this section is fundamental of some concepts. For convenience, the necessary definitions and theories used in the theory of fuzzy calculus are briefly given.

Definition 1. (Kaleva, 1987) A non-empty fuzzy set $v$ in a universe of discourse $X$ is described by a membership function $\mu_{v}: X \rightarrow[0,1]$, which associates with every point in $X$ a real number in $[0,1] \cdot \mu_{v}(x)$ is interpreted as the grade of membership of an element $\mathrm{x}$ in the fuzzy set $v$ for each $x \in X$.

Definition 2. (Kaleva, 1987) Let $\mathbb{R}$ stands to the set of all real numbers and $v$ is a non-empty set in $\mathbb{R}$. Then one says $v$ is a fuzzy number if it holds the following requirement:

1- $v$ is normal if there exists $x_{0} \in \mathbb{R}$ for which $v\left(x_{0}\right)=1$.

2- $v$ is convex, for each $x_{1}, x_{2} \in \mathbb{R}$ and $\lambda \in[0,1]$ which holds

that $v\left(\lambda x_{1}+(1-\lambda) x_{2}\right) \geq \min \left(v\left(x_{1}\right), v\left(x_{2}\right)\right)$.

3- $v$ is an upper semi-continuous, for each $x_{0} \in \mathbb{R}$,

$v\left(x_{0}\right) \geq \lim _{\mathrm{x} \rightarrow \mathrm{x}_{0}^{+}} v(x)$ and $v\left(x_{0}\right) \geq \lim _{\mathrm{x} \rightarrow \mathrm{x}_{0}^{-}} v(x)$.

4- The closure of $\operatorname{supp}(v)$ is compact.

For each $\sigma \in(0,1]$, set $[v]^{\sigma}=\{x \in \mathbb{R}: v(x) \geq \sigma\}$ and $[v]^{0}=$ $\overline{\{x \in \mathbb{R}: v(x)>0\}}$, where $\overline{\{\cdot\}}$ denote the closure of $\{\cdot\}$. Then, it can be easily proved that $v$ is a fuzzy number if and only if $[v]^{\sigma}$ is compact and convex set on $\mathbb{R}$ for all $\sigma \in[0,1]$ and $[v]^{1} \neq \varnothing$ (Goetschel \& Voxman, 1986). Further, let $v$ be a fuzzy number, then $[v]^{\sigma}=\left[v_{1}(\sigma), v_{2}(\sigma)\right]$. That is, $[v]^{\sigma}$ referred to the $\sigma$-cut representation or parametric form of a fuzzy number $v$. Where $v_{1}(\sigma)=\min \left\{x: x \in[v]^{\sigma}\right\}$ and $v_{2}(\sigma)=\max \left\{x: x \in[v]^{\sigma}\right\}$ for each $\sigma \in[0,1]$.

Theorem 3. (Goetschel \& Voxman, 1986) Suppose that $v_{1}, v_{2}:[0,1] \rightarrow \mathbb{R}$ which satisfies the following conditions;

1- $v_{1}$ is a bounded increasing function, and $v_{2}$ is a bounded decreasing function with $v_{1}(1) \leq v_{2}(1)$;

2- $v_{1}$ and $v_{2}$ are left-hand continuous functions at $\sigma=k$, for

all $k \in(0,1]$

3. $v_{1}$ and $v_{2}$ are right-hand continuous functions at $\sigma=0$.

Then, $v: \mathbb{R} \rightarrow[0,1]$ defined by $v(x)=\sup \left\{\sigma: v_{1}(\sigma) \leq x \leq\right.$ $\left.v_{2}(\sigma)\right\}$ is a fuzzy number and the parametric form is $\left[v_{1}(\sigma), v_{2}(\sigma)\right]$. Otherwise, the functions $v_{1}$ and $v_{2}$ satisfy the conditions above if $v: \mathbb{R} \rightarrow[0,1]$ is a fuzzy number with parametrisation $\left[v_{2}(\sigma), v_{1}(\sigma)\right]$.

Definition 4. (Puri \& Ralescu, 1983) For arbitrary fuzzy numbers $v$ and $w$ on $\mathbb{R}_{\mathrm{F}}$ we define the Hausdorff distance by the mapping $\mathrm{d}: \mathbb{R}_{\mathrm{F}} \times \mathbb{R}_{\mathrm{F}} \rightarrow \mathbb{R}^{+} \cup\{0\}$ such that

$$
\begin{gathered}
\mathrm{d}(v, w)=\sup _{0 \leq \sigma \leq 1} \max \left\{\left|v_{1}(\sigma)-w_{1}(\sigma)\right|, \mid v_{2}(\sigma)-\right. \\
\left.w_{2}(\sigma) \mid\right\},
\end{gathered}
$$

Definition 5. (Puri \& Ralescu, 1983) Let $f:[\mathrm{a}, \mathrm{b}] \rightarrow \mathbb{R}_{\mathrm{F}}$ be a fuzzy valued function. For fixed $x_{0} \in[\mathrm{a}, \mathrm{b}]$ and $\epsilon>$ 0 , there is $\delta>0$ with $\left|x-x_{0}\right|<\delta$ this implies $\mathrm{d}\left(f(x), f\left(x_{0}\right)\right)<\epsilon$, then we say that $f$ is continuous at $x_{0}$.

Definition 6. (Friedman et al., 1999) Suppose that $f:[\mathrm{a}, \mathrm{b}] \rightarrow \mathbb{R}_{\mathrm{F}}$ be a fuzzy valued function. For each partition $\mathrm{P}=\left\{x_{0}, x_{1}, \ldots, x_{n}\right\}$ of $[\mathrm{a}, \mathrm{b}]$ and $\zeta_{\mathrm{i}} \in\left[x_{i-1}, x_{i}\right], 1 \leq i \leq n$. Assume that $\mathrm{R}_{\mathrm{P}}=\sum_{i=1}^{n} x_{i}\left(\zeta_{i}\right)\left(x_{i}-x_{i-1}\right)$ and $\Delta=$ $\max _{1 \leq i \leq n}\left|x_{i-1}, x_{i}\right|$. The definite integral of $f(x)$ over $[\mathrm{a}, \mathrm{b}]$ is $\int_{\mathrm{a}}^{\mathrm{b}} f(x) d x=\lim _{\Delta \rightarrow 0} \mathrm{R}_{\mathrm{P}}$ such that the limit exists in $\left(\mathbb{R}_{\mathrm{F}}, \mathrm{d}\right)$. If the fuzzy function $f(x)$ is continuous in the metric $d$, its definite integral exists (Goetschel \& Voxman, 1986). Moreover,

$$
\left(\int_{a}^{b} f(x) d x\right)_{1 \sigma}=\int_{a}^{b} f_{1 \sigma}(x) d x
$$

and

$$
\left(\int_{a}^{b} f(x) d x\right)_{2 \sigma}=\int_{a}^{b} f_{2 \sigma}(x) d x .
$$

The fuzzy number $A$ is called the Hukuhara difference or "H-difference" of $v, w \in \mathbb{R}_{\mathrm{F}}$ and indicated by $v \ominus \mathrm{w}$, which is mean $\forall v, w \in \mathbb{R}_{\mathrm{F}}, \exists$ an element $\mathrm{A} \in \mathbb{R}_{\mathrm{F}}$ provided that $v=$ $w+A$. As well we referred always for Hukuhara difference by the sign " $\ominus$ " and let us mention that $v \ominus w \neq v+$ $(-1) w$

Definition 7. (Bede \& Gal, 2005) Let $f:[\mathrm{a}, \mathrm{b}] \rightarrow \mathbb{R}_{\mathrm{F}}$ and fixed $x_{0} \in[\mathrm{a}, \mathrm{b}]$. We say that $f$ is strongly generalized differentiable at $x_{0}$, if there exists an element $f^{\prime}\left(x_{0}\right) \in \mathbb{R}_{\mathrm{F}}$ such that either:

i- The H-differences $f\left(x_{0}+h\right) \ominus f\left(x_{0}\right), f\left(x_{0}\right) \ominus$

$f\left(x_{0}-h\right)$ exist and

$f^{\prime}\left(x_{0}\right)=\lim _{h \rightarrow 0^{+}} \frac{f\left(x_{0}+h\right) \ominus f\left(x_{0}\right)}{h}=\lim _{h \rightarrow 0^{+}} \frac{f\left(t_{0}\right) \ominus f\left(x_{0}-h\right)}{h}$,

for all $h>0$ sufficiently close to o, where the limits in a metric d, 
ii-The H-differences $f\left(x_{0}\right) \ominus f\left(x_{0}+h\right), f\left(x_{0}-h\right) \ominus f\left(x_{0}\right)$ exist and

$f^{\prime}\left(x_{0}\right)=\lim _{h \rightarrow 0^{+}} \frac{f\left(x_{0}\right) \ominus f\left(x_{0}+h\right)}{-h}=\lim _{h \rightarrow 0^{+}} \frac{f\left(x_{0}-h\right) \ominus f\left(x_{0}\right)}{-h}$,

for all $\mathrm{h}>0$ sufficiently close to $\mathrm{o}$, where the limits in a metric d.

Theorem 8. (Chalco-Cano \& Román-Flores, 2008) Assume that $v:[\mathrm{a}, \mathrm{b}] \rightarrow \mathbb{R}_{\mathrm{F}},[f(x)]^{\sigma}=\left[f_{1 \sigma}(x), f_{2 \sigma}(x)\right]$ for each $\sigma \in[0,1]$, then:

1- If $f$ is (1)-differentiable, then $f_{1 \sigma}$ and $f_{2 \sigma}$ are differentiable functions and $\left[\mathrm{D}_{1}^{1} f(x)\right]^{\sigma}=\left[f_{1 \sigma}^{\prime}(x), f_{2 \sigma}^{\prime}(x)\right]$,

2- If $f$ is (2)-differentiable, then $f_{1 \sigma}$ and $f_{2 \sigma}$ are differentiable functions and $\left[\mathrm{D}_{2}^{1} f(x)\right]^{\sigma}=\left[f_{2 \sigma}^{\prime}(x), f_{1 \sigma}^{\prime}(x)\right]$.

\section{FUZZY VOLTERRA INTEGRODIFFERENTIL EQUATIONS}

This section aims to provide and discuss the fuzzy approximate solution of the following fuzzy Volterra integro-differential equations with the fuzzy initial conditions under the concept of strongly generalised differentiability

$$
u^{\prime}(x)=\varphi(x)+\lambda \int_{0}^{x} g(x, t) u(t) d t,
$$

subject to the initial condition

$$
u(0)=u_{0},
$$

where $\lambda$ is a positive parameter, $g(x, t)$ is a function, called an arbitrary kernel function and $\varphi$ is a continuous function of $x$. To solve Eq. (1) and (2), we write the given fuzzy equation in the parametric forms as follows:

$$
\begin{aligned}
& u_{1 \sigma}^{\prime}(x)=\varphi_{1 \sigma}(x)+\lambda \int_{0}^{x} G_{1 \sigma}(x, t, u(t)) d t, \\
& u_{2 \sigma}^{\prime}(x)=\varphi_{2 \sigma}(x)+\lambda \int_{0}^{x} G_{2 \sigma}(x, t, u(t)) d t,
\end{aligned}
$$

with $u_{1 \sigma}(0)=u_{01 \sigma}$ and $u_{2 \sigma}(0)=u_{02 \sigma}$,

Where,

$$
G_{1 \sigma}(x, t, u(t))= \begin{cases}g(x, t) u_{1 \sigma}(t), & g(x, t) \geq 0 \\ g(x, t) u_{2 \sigma}(t), & g(x, t)<0,\end{cases}
$$

and

$$
G_{2 \sigma}(x, t, u(t))= \begin{cases}g(x, t) u_{2 \sigma}(t), & g(x, t) \geq 0 \\ g(x, t) u_{1 \sigma}(t), & g(x, t)<0\end{cases}
$$

\section{DESCRIPTION OF THE RPS METHOD}

In this section, we describe the RPS scheme to provide the approximate solution for Eq. (1) and (2). To do so, we assume that the solution of Eq. (3) has the following power series expansion about the initial point $x_{0}=0$ :

$$
\begin{aligned}
& u_{1 \sigma}(x)=\sum_{n=0}^{\infty} a_{n} x^{n}, \\
& u_{2 \sigma}(x)=\sum_{n=0}^{\infty} b_{n} x^{n} .
\end{aligned}
$$

Applying the initial conditions of Eq. (3) in the series solutions in Eq. (4), we get that $u_{1 \sigma}(0)=u_{01 \sigma}=a_{0}$ and $u_{2 \sigma}(0)=u_{02 \sigma}=b_{0}$, and hence we can approximate the solutions $u_{1 \sigma}(x)$ and $u_{2 \sigma}(x)$ by the $m^{\text {th }}$-truncated series as:

$$
\begin{aligned}
& u_{m, 1 \sigma}(x)=a_{0}+\sum_{n=1}^{m} a_{n} x^{n}, \\
& u_{m, 2 \sigma}(x)=b_{0}+\sum_{n=1}^{m} b_{n} x^{n} .
\end{aligned}
$$

According to the RPS approach (Moaddy et al., 2015; Gumah et al., 2018; Komashynska et al., 2016; Hasan et al., 2019; Komashynska et al., 2016) in finding the unknown constants $a_{n}$ and $b_{n}, n=1,2, \ldots, m$, we define the $m^{t h}$-residual functions as the following:

$$
\begin{aligned}
\operatorname{Res}_{m, 1 \sigma}(x) & =u_{m, 1 \sigma}^{\prime}(x)-\varphi_{1 \sigma}(x) \\
& -\lambda \int_{0}^{x} g(x, t) u_{m, 1 \sigma}(t) d t \\
\operatorname{Res}_{m, 2 \sigma}(x) & =u_{m, 2 \sigma}^{\prime}(x)-\varphi_{2 \sigma}(x) \\
& -\lambda \int_{0}^{x} g(x, t) u_{m, 2 \sigma}(t) d t .
\end{aligned}
$$

Now, to obtain the unknown constants $a_{1}$ and $b_{1}$, substitute $u_{1,1 \sigma}(x)=a_{0}+a_{1} x$ and $u_{1,2 \sigma}(x)=b_{0}+b_{1} x$, into the first residual equation as follows

$$
\begin{gathered}
\operatorname{Res}_{1,1 \sigma}(x)=a_{1}-\varphi_{1 \sigma}(x)-\lambda \int_{0}^{x} g(x, t)\left(a_{0}+a_{1} t\right) d t \\
\operatorname{Res}_{1,2 \sigma}(x)=b_{1}-\varphi_{2 \sigma}(x)-\lambda \int_{0}^{x} g(x, t)\left(b_{0}+b_{1} t\right) d t
\end{gathered}
$$

Then, by using the facts $\operatorname{Res}_{1,1 \sigma}(0)=\operatorname{Res}_{1,2 \sigma}(0)=0$, it yields

$$
\begin{aligned}
& a_{1}=\left(\frac{\varphi_{1 \sigma}(x)+\lambda a_{0} \int_{0}^{x} g(x, t) d t}{1-\lambda \int_{0}^{x} \operatorname{tg}(x, t) d t}\right)_{x=0}, \\
& b_{1}=\left(\frac{\varphi_{2 \sigma}(x)+\lambda b_{0} \int_{0}^{x} g(x, t) d t}{1-\lambda \int_{0}^{x} \operatorname{tg}(x, t) d t}\right)_{x=0} .
\end{aligned}
$$

Again, to find $a_{2}$ and $b_{2}$, substitute

$u_{2,1 \sigma}(x)=a_{0}+a_{1} x+a_{2} x^{2}$ and $u_{2,2 \sigma}(x)=b_{0}+b_{1} x+b_{2} x^{2}$ into the second residual equation as 


$$
\begin{aligned}
\operatorname{Res}_{2,1 \sigma}(x)=\left(a_{1}+\right. & \left.2 a_{2} x\right)-\varphi_{1 \sigma}(x) \\
& -\lambda \int_{0}^{x} g(x, t)\left(a_{0}+a_{1} t+a_{2} t^{2}\right) d t \\
\operatorname{Res}_{2,2 \sigma}(x)=\left(b_{1}+\right. & \left.2 b_{2} x\right)-\varphi_{2 \sigma}(x) \\
& -\lambda \int_{0}^{x} g(x, t)\left(b_{0}+b_{1} t+b_{2} t^{2}\right) d t .
\end{aligned}
$$

Using the fact $\frac{d}{d x} \operatorname{Res}_{2,1 \sigma}(0)=\frac{d}{d x} \operatorname{Res}_{2,2 \sigma}(0)=0$, we get that

$$
\begin{aligned}
& a_{2}=\left(\frac{\varphi_{1 \sigma}^{\prime}(x)+\lambda a_{0} \int_{0}^{x} \frac{\partial}{\partial x} g(x, t) d t+\lambda a_{1} \int_{0}^{x} \frac{\partial}{\partial x} g(x, t) t d t}{2-\lambda \int_{0}^{x} \frac{\partial}{\partial x} g(x, t) t^{2} d t}\right)_{x=0}, \\
& b_{2}=\left(\frac{\varphi_{2 \sigma}^{\prime}(x)+\lambda b_{0} \int_{0}^{x} \frac{\partial}{\partial x} g(x, t) d t+\lambda b_{1} \int_{0}^{x} \frac{\partial}{\partial x} g(x, t) t d t}{2-\lambda \int_{0}^{x} \frac{\partial}{\partial x} g(x, t) t^{2} d t}\right)_{x=0} .
\end{aligned}
$$

Likewise, for $m=3$, and by using the facts $\frac{d^{2}}{d x^{2}} \operatorname{Res}_{3,1 \sigma}(0)=\frac{d^{2}}{d x^{2}} \operatorname{Res}_{3,2 \sigma}(0)=0$, we can find $a_{3}$ and $b_{3}$ as

$$
\begin{gathered}
a_{3}=\left(\frac { 1 } { 6 - \lambda \int _ { 0 } ^ { \mathrm { x } } \frac { \partial ^ { 2 } } { \partial x ^ { 2 } } g ( \mathrm { x } , \mathrm { t } ) t ^ { 3 } d t } \left(\varphi_{1 \sigma}^{\prime \prime}(x)+\lambda a_{0} \int_{0}^{\mathrm{x}} g(x, t) d t+\right.\right. \\
\left.\left.\lambda a_{1} \int_{0}^{x} \frac{\partial^{2}}{\partial x^{2}} g(x, t) t d t+\lambda a_{2} \int_{0}^{x} \frac{\partial^{2}}{\partial x^{2}} g(x, t) t^{2} d t\right)\right)_{x=0}, \\
b_{3}=\left(\frac { 1 } { 6 - \lambda \int _ { 0 } ^ { \mathrm { x } } \frac { \partial ^ { 2 } } { \partial x ^ { 2 } } g ( \mathrm { x } , \mathrm { t } ) t ^ { 3 } d t } \left(\varphi_{2 \sigma}^{\prime \prime}(x)+\lambda b_{0} \int_{0}^{\mathrm{x}} g(x, t) d t+\right.\right. \\
\left.\left.\lambda b_{1} \int_{0}^{x} \frac{\partial^{2}}{\partial x^{2}} g(x, t) t d t+\lambda b_{2} \int_{0}^{x} \frac{\partial^{2}}{\partial x^{2}} g(x, t) t^{2} d t\right)\right)_{x=0} .
\end{gathered}
$$

\section{ILLUSTRATIVE EXAMPLE}

In the current section, we illustrate the efficiency and applicably of the proposed method by providing the numerical solutions of the following fuzzy Volterra integro-differential equation

$$
\begin{aligned}
& u^{\prime}(x)=\alpha\left(e^{x}(2-x)-1\right)+\int_{0}^{x} t u(t) d t, x \in[0,1], 0< \\
& t \leq x, \\
& \text { with the fuzzy initial conditions } \\
& u(0)=\alpha,
\end{aligned}
$$

where the $\sigma$-cut representation of $\alpha$ is $[0.5+0.5 \sigma, 2-\sigma], 0 \leq$ $\sigma \leq 1$

The exact solution is given by $u(x)=[0.5+0.5 \sigma, 2-\sigma] e^{t}$.

Thus, Eq. (11) can be written in the parametric forms as follows:

$$
u_{1 \sigma}^{\prime}(x)=(0.5+0.5 \sigma)\left(e^{t}(2-t)-\right.
$$$$
\text { 1) } \int_{0}^{x} t u_{1 \sigma}(t) d t
$$

$$
\begin{array}{r}
u_{2 \sigma}^{\prime}(x)=(2-\sigma)\left(e^{t}(2-t)-1\right) \\
+\int_{0}^{x} t u_{2 \sigma}(t) d t,
\end{array}
$$

Subject to $u_{1 \sigma}(0)=0.5+0.5 \sigma$

and $u_{2 \sigma}(0)=2-\sigma$.

To show the accuracy of the RPS technique, the exact and approximate solutions are compared in Table 1 . The results obtained by the RPS method show that the exact solutions are in good agreement with approximate solutions at $\sigma=1$ and $n=8$.

Table 1: Numerical results at $\sigma=1$ and $n=8$.

\begin{tabular}{|c|c|c|c|}
\hline$x_{i}$ & Exact & Approximation & Absolute error \\
\hline 0.1 & 1.105170918 & 1.10517091807 & $2.88657 \times 10^{-15}$ \\
\hline 0.3 & 1.349858807 & 1.34985880752 & $5.59139 \times 10^{-11}$ \\
\hline 0.5 & 1.648721271 & 1.64872126503 & $5.66416 \times 10^{-9}$ \\
\hline 0.7 & 2.013752707 & 2.01375258795 & $1.19513 \times 10^{-7}$ \\
\hline 0.9 & 2.459603111 & 2.45960193894 & $1.17221 \times 10^{-6}$ \\
\hline
\end{tabular}

To show the fuzzy behaviour of the proposed algorithm, the lower and upper approximate solutions are plotted in Figures 1, 2 and 3 for $t=0,0.25$ and 0.75 with $n=5$, and $\sigma \in[0,1]$. From these figures, it can be noted that the solutions are represented in the shapes of symmetric triangular fuzzy along the direction of $\sigma$ and with different values of $t$.

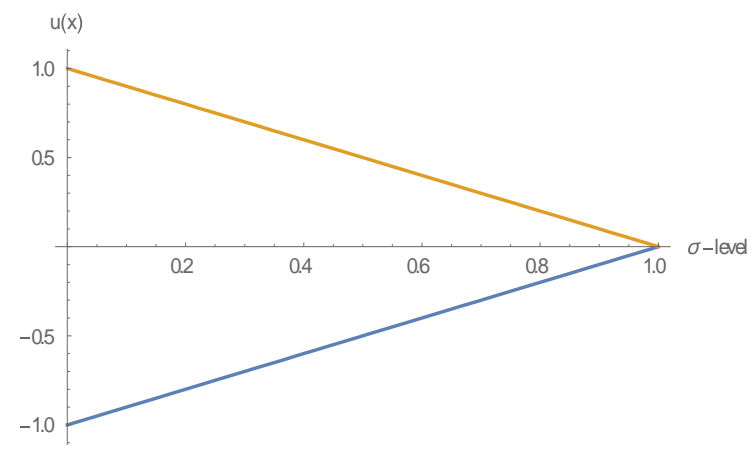

Figure 1: Solution plot for $\sigma \in[0,1], t=0, n=5$. 


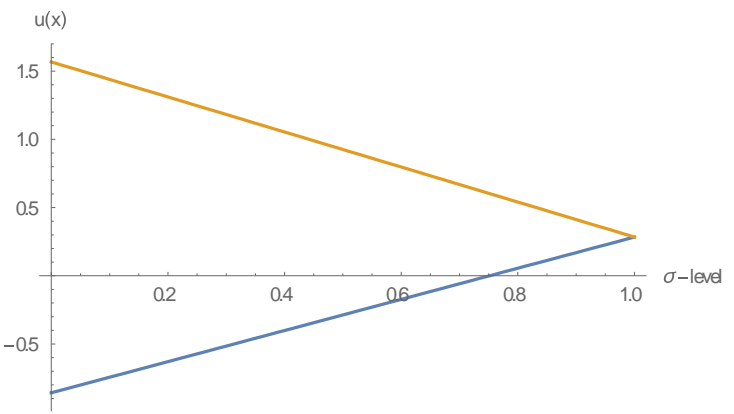

Figure 2: Solution plot for $\sigma \in[0,1], t=0.25, n=5$.

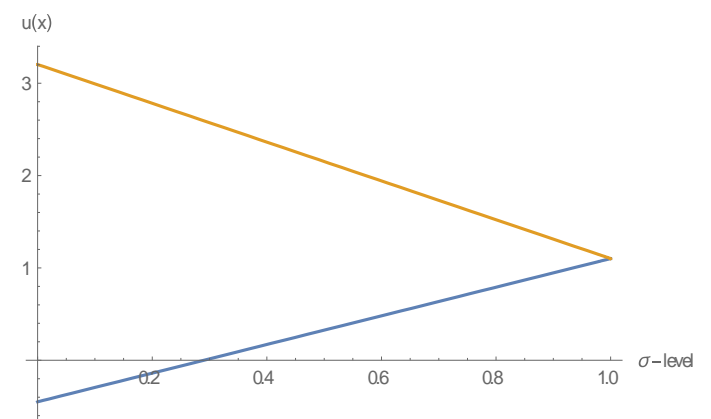

Figure 3: Solution plot for $\sigma \in[0,1], t=0.75, n=5$.

\section{CONCLUSION}

In this paper, the RPS scheme has been applied to provide the approximate solution for fuzzy Volterra IDEs with appropriate fuzzy initial conditions under strongly generalised $\mathrm{H}$ differentiability. This method can be applied directly to the given problem by choosing an appropriate initial guess approximation. Numerical results have shown the performance and reliability of the present approach. The results indicate that the RPS method is very efficient and powerful in solving fuzzy differential equations with fewer calculations and time.

\section{ACKNOWLEDGEMENT}

This research was financially supported by the Universiti

Kebangsaan Malaysia (UKM) under the grant (GSP/1/2015/SGo4/UKM/01/1). 


\section{REFERENCES}

Abbasbandy, S. \& Hashemi, M. 2012, 'A series solution of fuzzy integro-differential equations', Journal of Fuzzy Set Valued Analysis, vol. 2012, pp. 1-17.

Abbasbandy, S. \& Hashemi, M. S. 2010, 'Fuzzy integrodifferential equations: formulation and solution using the variational iteration method', Nonlinear Science Letters $A$, vol. 1, no. 4, pp. 413-418.

Abu Arqub, O. 2017, 'Adaptation of reproducing kernel algorithm for solving fuzzy Fredholm-Volterra integrodifferential equations', Neural Computing and Applications, vol. 28, no. 7, pp. 1591-1610.

Abu Arqub, O. \& Al-Smadi, M. 2018, 'Atangana-Baleanu fractional approach to the solutions of Bagley-Torvik and Painlevé equations in Hilbert space', Chaos, Solitons \& Fractals, vol. 117, pp. 161-167.

Abu Arqub, O. \& Al-Smadi, M. 2018, 'Numerical algorithm for solving time-fractional partial integrodifferential equations subject to initial and Dirichlet boundary conditions', Numerical Methods for Partial Differential Equations, vol. 34, no. 5, pp. 1577-1597.

Abu Arqub, O., AL-Smadi, M., Momani, S. \& Hayat, T. 2016, 'Numerical solutions of fuzzy differential equations using reproducing kernel Hilbert space method', Soft Computing, vol. 20, no. 8, pp. 3283-3302.

Abu Arqub, O., Odibat, Z. \& Al-Smadi, M. 2018, 'Numerical solutions of time-fractional partial integrodifferential equations of Robin functions types in Hilbert space with error bounds and error estimates', Nonlinear Dynamics, vol. 94, no. 3, pp. 1819-1834.

Abu-Gdairi, R., Al-Smadi, M. \& Gumah, G. 2015, 'An Expansion Iterative Technique for Handling Fractional Differential Equations Using Fractional Power Series Scheme', Journal of Mathematics and Statistics, vol. 11 no. 2, pp. 29-38.

Al-Smadi, M. 2018, 'Simplified iterative reproducing kernel method for handling time-fractional BVPs with error estimation', Ain Shams Engineering Journal, vol. 9, no 4, pp. 2517-2525.

Al-smadi, M. 2019, 'Reliable Numerical Algorithm for Handling Fuzzy Integral Equations of Second Kind in Hilbert Spaces', Filomat, vol. 33, no. 2, pp. 583-597.
Al-Smadi, M. \& Arqub, O. A. 2019, 'Computational algorithm for solving fredholm time-fractional partial integrodifferential equations of dirichlet functions type with error estimates', Applied Mathematics and Computation, 2, vol. 342, pp. 280-294.

Al-Smadi, M. et al. 2017, 'Numerical Multistep Approach for Solving Fractional Partial Differential Equations', International Journal of Computational Methods, vol. 14, no. 3, 1750029, 15p.

Altawallbeh, Z., Al-Smadi, M., Komashynska, I. \& Ateiwi, A. 2018, 'Numerical Solutions of Fractional Systems of Two-Point BVPs by Using the Iterative Reproducing Kernel Algorithm', Ukrainian Mathematical Journal, vol. 70, no. 5, pp. 687-701.

Arqub, O. A., Al-Smadi, M., Momani, S. \& Hayat, T. 2017, 'Application of reproducing kernel algorithm for solving second-order, two-point fuzzy boundary value problems', Soft Computing, vol. 21, no. 23, pp. 7191-7206.

Bede, B. \& Gal, S. G. 2005, 'Generalizations of the differentiability of fuzzy-number-valued functions with applications to fuzzy differential equations', Fuzzy Sets and Systems, vol. 151, no. 3, pp. 581-599.

Chalco-Cano, Y. \& Román-Flores, H. 2008, 'On new solutions of fuzzy differential equations', Chaos, Solitons \& Fractals, vol. 38, no. 1, pp. 112-119.

Freihet, A. et al. 2019, 'Construction of fractional power series solutions to fractional stiff system using residual functions algorithm', Advances in Difference Equations, vol. 2019, no. 1, p. 95.

Friedman, M., Ma, M. \& Kandel, A. 1999, 'Numerical solutions of fuzzy differential and integral equations', Fuzzy Sets and Systems, vol. 106, no. 1, pp. 35-48.

Goetschel, R. \& Voxman, W. 1986, 'Elementary fuzzy calculus', Fuzzy Sets and Systems, vol. 18, no. 1, pp. 31-43. Gumah, G., Moaddy, K., Al-Smadi, M. \& Hashim, I. 2016, 'Solutions to Uncertain Volterra Integral Equations by Fitted Reproducing Kernel Hilbert Space Method', Journal of Function Spaces, volume 2016.

Gumah, G. N., Naser, M. F. M., Al-Smadi, M. \& Al-Omari, S. K. 2018, 'Application of reproducing kernel Hilbert space method for solving second-order fuzzy Volterra integro-differential equations', Advances in Difference Equations, vol. 2018, no. 1, p. 475. 
Hasan, S., Al-Smadi, M., Freihet, A. \& Momani, S. 2019, 'Two computational approaches for solving a fractional obstacle system in Hilbert space', Advances in Difference Equations, vol. 2019, no. 1, p. 55 .

Kaleva, O. 1987, 'Fuzzy differential equations', Fuzzy Sets and Systems, vol. 24, no. 3, pp. 301-317.

Komashynska, I., Al-Smadi, M., Abu Arqub, O. \& Momani, S. 2016, 'An Efficient Analytical Method for Solving Singular Initial Value Problems of Nonlinear Systems', Applied Mathematics \& Information Sciences, vol. 10, no. 2, pp. 647656.

Komashynska, I., Al-Smadi, M., Ateiwi, A. \& Al-Obaidy, S. 2016, 'Approximate Analytical Solution by Residual Power Series Method for System of Fredholm Integral Equations', Applied Mathematics \& Information Sciences, vol. 10, no. 3, pp. 975-985.

Moaddy, K., AL-Smadi, M. \& Hashim, I. 2015, 'A Novel Representation of the Exact Solution for Differential Algebraic Equations System Using Residual Power-Series Method', Discrete Dynamics in Nature and Society, vol. 2015, pp. 1-12.

Puri, M. L. \& Ralescu, D. A. 1983, 'Differentials of fuzzy functions', Journal of Mathematical Analysis and Applications, vol. 91, no. 2, pp. 552-558.

Saadeh, R. et al 2016, 'Numerical investigation for solving two-point fuzzy boundary value problems by reproducing kernel approach', Applied Mathematics and Information Sciences, vol. 10, no. 6, pp. 2117-2129. 